\title{
Enhancement of microcavity polariton relaxation under confinement
}

\author{
T. K. Paraïso, ${ }^{1 *}$ D. Sarchi, ${ }^{2}$ G. Nardin,,${ }^{1}$ R. Cerna,${ }^{1}$ Y. Leger, ${ }^{1}$ B. Pietka, ${ }^{1}$ M. Richard,,${ }^{1,}$ O. El Daïf, ${ }^{1, *}$ F. Morier-Genoud, ${ }^{1}$ \\ V. Savona, ${ }^{2}$ and B. Deveaud-Plédran ${ }^{1}$ \\ ${ }^{1}$ Laboratory of Quantum Optoelectronics, EPFL, CH-1015, Lausanne, Switzerland \\ ${ }^{2}$ Institute of Theoretical Physics, EPFL, CH-1015, Lausanne, Switzerland
}

(Received 1 September 2008; revised manuscript received 28 November 2008; published 29 January 2009)

\begin{abstract}
We experimentally investigate the relaxation of spatially confined microcavity polaritons. We measure the time- and energy-resolved photoluminescence under resonant excitation and in the low-density regime. In this way, we have access to the time evolution of the energy distribution of the polariton population. We show that, when one confined level is resonantly excited, after an initial transient, the population of the confined levels is thermally distributed. The reported efficiency of the relaxation process strongly depends on the confinement size. These experimental findings are well reproduced by a theoretical model accounting for the coupling between the confined states and a bath of acoustic phonons. Our results thus suggest that the phonon-mediated relaxation mechanisms are enhanced in the presence of spatial confinement.
\end{abstract}

DOI: 10.1103/PhysRevB.79.045319

PACS number(s): 71.36.+c, 71.35.Gg, 63.20.kk

\section{INTRODUCTION}

Microcavity (MC) polaritons are two-dimensional quasiparticles resulting from the strong coupling between excitons and electromagnetic modes in semiconductor microcavities. Owing to their bosonic behavior and their very light effective mass $\left(10^{-5}\right.$ times the free-electron mass), MC polaritons are the ideal tool for investigating quantum phenomena in solidstate systems. During the last 10 years a large number of studies have been devoted to the study of the properties of MC polaritons. ${ }^{1-6}$ This activity lead to the observation of parametric processes, ${ }^{6}$ polariton lasing, ${ }^{4,5}$ and the demonstration of polariton Bose-Einstein condensation (BEC). ${ }^{1-3}$ This latter achievement makes MC polaritons the most promising system to study many fundamental aspects of the BEC physics and superfluidity ${ }^{7,8}$ in solid-state systems due to the ease of optical measurements.

To address these challenges, it would be important to study polariton BEC in a regime very close to thermal equilibrium. Indeed, theory predicts the manifestation of the superfluid behavior at lower densities and temperatures than the ones commonly explored..$^{9,10}$ In planar microcavities, due to the short radiative lifetime of polaritons and the very inefficient mechanisms of energy relaxation and thermalization, the deviations from equilibrium are very important. Indeed, in planar microcavities, thermalization is only possible in the regime of high polariton densities, where the polariton-polariton scattering becomes efficient enough. ${ }^{1-3}$ In the low-density regime, where the only mechanism of thermalization is the energy exchange between polaritons and phonons or free charges, polaritons do not thermalize. In this density regime, the relaxation can be enhanced only by raising the temperature or by injecting free carriers in the system. ${ }^{11-13}$

Recently, it has been suggested that the presence of spatial confinement could have an impact on the thermalization process. ${ }^{14}$ This is confirmed by pioneering experimental observations in micropillars, where the spontaneous quantum degeneracy of microcavity polaritons seems to be reached at lower excitation densities than in planar microcavities. ${ }^{15}$ In order to clarify this point, a detailed investigation of the efficiency of relaxation and thermalization of polaritons in spatially confined structures is required.

In this paper, we demonstrate the efficiency of polariton relaxation and thermalization under lateral confinement in the low-density regime. For this purpose, we investigated a GaAs/AlAs microcavity where we engineered high-quality traps capable of confining polaritons on the micrometer scale. ${ }^{16}$ By means of photoluminescence excitation (PLE) and time-resolved spectroscopy, we measured the time evolution of the polariton energy distribution for different excitation energies. We demonstrate that the spatial confinement significantly enhances polariton relaxation because of the lift of the momentum selection rule. This leads to efficient thermalization within the localized polariton states. On the other hand, we show that the energy relaxation between delocalized and localized polariton states is inefficient. Experimental findings are reproduced well by a theoretical model that accounts for the confining potential and for the coupling between polaritons and a bath of acoustic phonons in a semiclassical framework. The good agreement between theoretical predictions and experimental results suggests that the relaxation mechanism is dominated by phonon-assisted scattering processes, whose efficiency is enhanced by polariton localization.

The paper is organized as follows. The system and its spectral properties are described in Sec. II. In Sec. III, we present the experimental evidence of the thermalization of confined polaritons. Section IV is dedicated to the experimental study of the thermalization dynamics. The theoretical model is exposed in Sec. V, while the comparison between the experimental results and the theoretical predictions is discussed in Sec. VI. A general conclusion is given in Sec. VII.

\section{SAMPLE AND SPECTRAL PROPERTIES}

The investigated sample is exhaustively described in Refs. 16 and 17 . It consists of a $\lambda$ GaAs/AlAs microcavity containing a single $8 \mathrm{~nm}$ InGaAs quantum well (QW) and surrounded by two distributed Bragg reflectors made of 21 (top) 
(a)

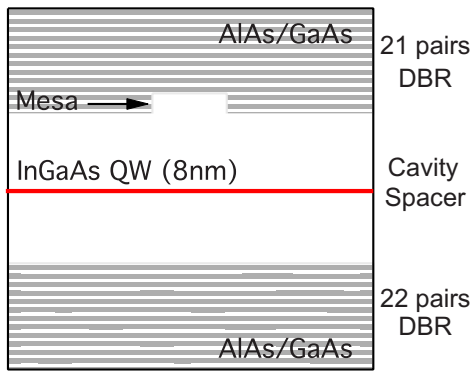

(b)

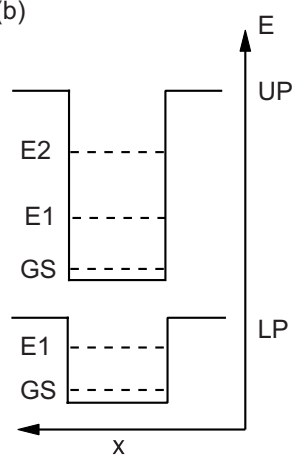

FIG. 1. (Color online) (a) Scheme of a $\lambda$ microcavity with a mesa. Within a limited area, the thickness of the spacer is increased, resulting in an effective potential well for the cavity modes. This gives rise to a confining potential for polaritons. (b) Scheme of the potential trap induced by the mesa. The resulting discrete energy levels of the confined upper polariton (UP) and lower polariton (LP) are also sketched [ground state (GS) and excited states E1 and E2].

and 22 (bottom) AlAs/GaAs $\lambda / 4$ layer pairs. The whole structure was grown with a wedge, allowing one to tune the detuning $\delta=E_{C}-E_{X}$ between the cavity energy $E_{C}$ and the exciton energy $E_{X}$ by changing position on the sample.

We designed local cylindrical extensions of the cavity length (mesas), which constitute potential wells for the electromagnetic modes [Fig. 1(a)]. This results in effective potential wells for polaritons because of the strong coupling between the cavity photon modes and the quantum well excitons. In Fig. 1(b), we sketch the potential wells and the corresponding discrete energy levels for both upper (UP) and lower (LP) polaritons. The differences in the potential depths and in the energy spacing between the levels are due to the different photonic contents.

The demonstration of the strong-coupling regime and of the efficient trapping of polaritons in mesas of different diameters is reported in Refs. 16 and 17. We measured Rabi splittings of $3.5 \mathrm{meV}$ in the planar cavity and of $3.35 \mathrm{meV}$ in the mesas.

For all experiments discussed in the present paper, the sample was cooled down to $5 \mathrm{~K}$ in a liquid-helium continuous-flow cryostat. We studied three mesas of 3, 9, and $20 \mu \mathrm{m}$ diameter, all at slightly negative detuning $\delta \simeq-0.6 \mathrm{meV}$ for the lowest-energy state.

The excitation beam was focused on the back side of the sample. We used a microscope objective with a large numerical aperture $(\mathrm{NA}=0.5)$ and centered the 3 - $\mu \mathrm{m}$-diameter laser spot on the mesa in order to ensure a maximal mode matching between the excitation and any localized polariton state. The detection was done at the front side of the sample using a microscope objective with the same numerical aperture. The collected luminescence was sent to an imaging spectrometer with $100 \mu \mathrm{eV}$ resolution.

The spatially resolved spectrum of a 9 - $\mu$ m-diameter mesa under continuous-wave nonresonant excitation is displayed in Fig. 2. The energy ranges of the UP and LP delocalized [two-dimensional (2D)] and localized [zero-dimensional (0D)] polaritons are separated by horizontal lines. In the regions labeled LPOD and UPOD, both lower and upper polari-

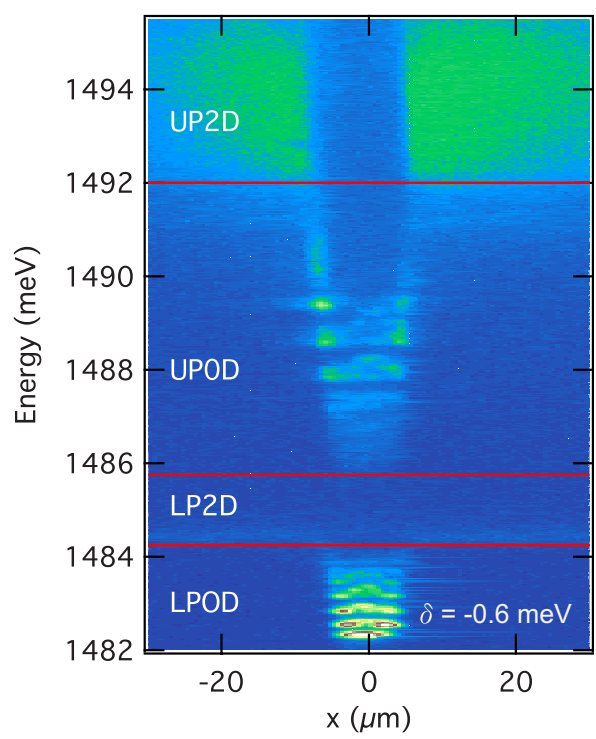

FIG. 2. (Color online) Spatially resolved luminescence spectrum of a 9- $\mu$ m-diameter mesa under nonresonant excitation (1570 $\mathrm{meV})$. One can notice the presence of discrete energy levels corresponding to states localized inside the mesa $(-5$ to $-5 \mu \mathrm{m})$. The strong emission from the localized lower polariton levels indicates that free carriers efficiently relax down to the low-energy levels. The horizontal lines are guides for the eyes separating the different polariton energy ranges. The detuning $\delta=-0.6 \mathrm{meV}$ is calculated for the ground state which is here $42 \%$ excitonic and $58 \%$ photonic.

tons are confined by the mesa. As sketched in Fig. 1(b), the luminescence spectrum of $0 \mathrm{D}$ polaritons is discrete, and for both LPOD and UPOD we can identify one ground state and some excited states. In this paper, the term "ground state" is used to refer to the ground state of the LPOD.

In the LP2D and UP2D regions, the luminescence is mainly detected outside the mesa. Indeed, at these energies polariton states are delocalized. The wave functions of delocalized states vanish above the mesa, and hence the luminescence intensity of $2 \mathrm{D}$ polaritons is very low at this position. The detuning, $\delta=-0.6 \mathrm{meV}$ for the ground state, corresponds to a very positive detuning for the cavity 2D states. The LP2D is therefore nearly purely excitonic and the luminescence from this region is strongly suppressed.

Under nonresonant excitation, free carriers are scattered by optical phonons to the nonradiative region of the exciton dispersion curve. Multiple scattering with acoustic phonons brings the excitons down to the radiative region, where they strongly couple to light and finally form polaritons. ${ }^{18}$ The full process is governed by the slow relaxation of excitons in the nonradiative region, because the radiative lifetime of polaritons is much shorter than the exciton lifetime. Therefore, the relaxation dynamics between confined polariton states can be more likely characterized by means of resonant excitation experiments, such as PLE.

\section{THERMALIZATION OF OD POLARITONS}

We performed two types of PLE experiments. Both consisted in detecting the luminescence spectrum while exciting 
resonantly the confined states. In one case, the excitation source was a continuous tunable Ti:sapphire laser (cw-PLE) in order to perform precise excitation of single-energy states of the system. In the other case, the excitation source was a pulsed Ti:sapphire laser of $500 \mu \mathrm{eV}$ spectral full width at half maximum (FWHM) (p-PLE). It allowed us to take the relaxation dynamics into account and to study the system by resonant time-resolved photoluminescence (TRPL) (Sec. IV).

An image plot of the time-integrated p-PLE is shown in Fig. 3. The excitation energies are displayed along the horizontal axis whereas the detected luminescence spectra are displayed vertically. On the diagonal, the emission energy is equal to the excitation energy. We make four key observations:

(i) When exciting the LP0D (from 1482 to $1484.5 \mathrm{meV}$ ), polaritons redistribute within LP0D states toward both lower and higher energies. This indicates the presence of efficient interactions with a thermal bath.

(ii) When the excitation is resonant with the UP0D (from 1485.5 to $1490 \mathrm{meV}$ ), the relaxation to LP0D states is very efficient. We do not observe significant polariton redistribution within the UPOD states.

(iii) When exciting the UPOD, the relative intensities of the LP0D states suggest that thermalization occurs within the LP0D states.

(iv) Contrary to (ii), when the excitation is resonant with the 2D polaritons, the relaxation to LPOD states is inefficient.

We give an interpretation of (i) and (iii) in terms of thermalization whereas (ii) and (iv) are discussed with considerations on the polariton dimensionality.

\section{A. Thermalization in mesas}

The p-PLE experiments were carried out in the lowdensity regime, where polariton-polariton interactions are negligible. Therefore, we attribute the relaxation to polariton-acoustic phonon scattering. Observations (i) and (iii) are clear evidence of efficient thermalization within the LP0D states.

To study in detail the thermalization within LP0D, we focus on the case in which we excite resonantly a UP0D state (1487 meV). In Fig. 4, we display the time-integrated emission per state, as a function of the energy of the state. This quantity is obtained from the LPOD luminescence spectrum by integrating the luminescence intensity of each peak. It is equal to the population per state weighted by the photonic content of the state.

The data are in good agreement with Boltzmann distributions, from which we can extract effective temperatures. The photonic content of the states was taken into account in the Boltzmann distributions by applying an energy-dependent correction factor, which explains the nonexponential behavior of the fitting curves.

We obtain effective temperatures of $15 \mathrm{~K}$ for the $\emptyset 3 \mu \mathrm{m}$ mesa and $40 \mathrm{~K}$ for the $\varnothing 9 \mu \mathrm{m}$ mesa. The emission of the $\varnothing$ $20 \mu \mathrm{m}$ mesa could not be fitted by a thermal distribution.

The efficiency of thermalization depends on the difference between the polariton-phonon scattering time $\tau_{\mathrm{ph}}$ and the po-
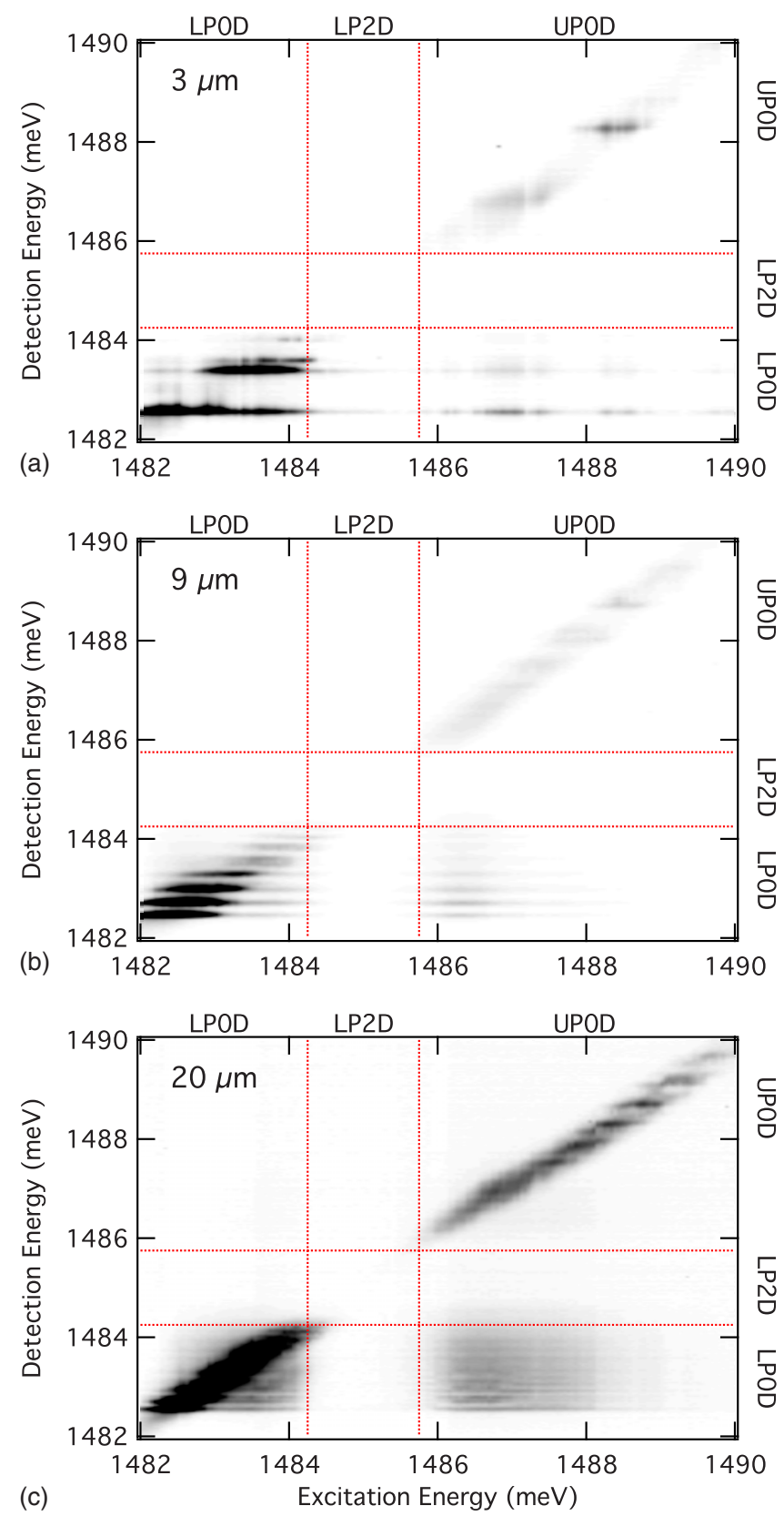

FIG. 3. (Color online) Image plots of p-PLE experiments on (a) 3- $\mu \mathrm{m}-$, (b) $9-\mu \mathrm{m}-$, and (c) 20- $\mu \mathrm{m}$-diameter mesas. The dotted lines are separations between the LPOD, LP2D, and UP0D energy ranges. The relaxation down to the lowest-energy state is favored and suggests the presence of a thermalization process. The weak signal between the dotted lines indicates a poor coupling between polaritonic states of different dimensionalities. The resolution is $100 \mu \mathrm{eV}$ on both axes.

lariton radiative lifetime $\tau_{r}$. There are three possible situations: ${ }^{19}$

(a) $\tau_{r} \gg \tau_{\mathrm{ph}}$ : During their lifetime, polaritons scatter many times with phonons. It is possible to define a temperature. This temperature is close to the lattice temperature.

(b) $\tau_{r} \geq \tau_{\mathrm{ph}}$ : The two time scales are comparable. Polaritons thermalize at a higher temperature than the lattice. 


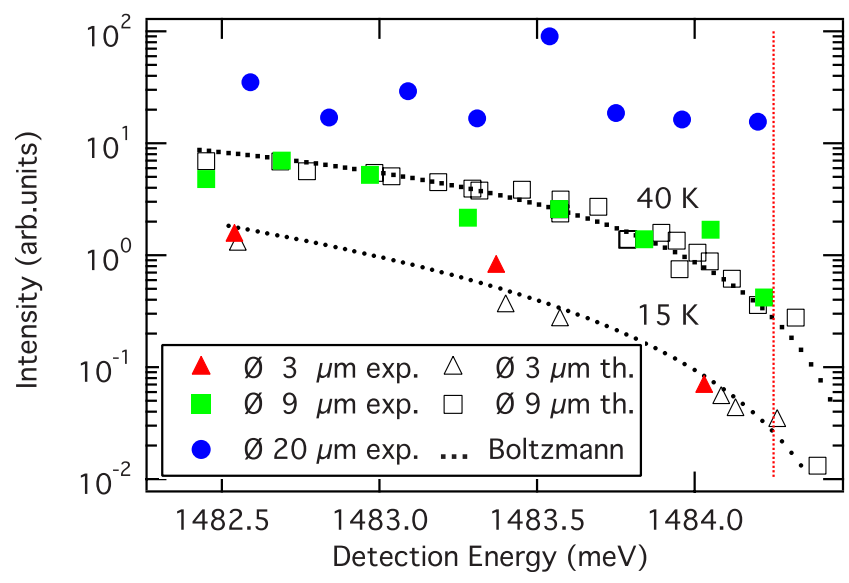

FIG. 4. (Color online) Integrated luminescence intensities of the LP0D states for an excitation in the UP0D $(1487 \mathrm{meV})$ for the three sizes of mesas. Experimental data (filled symbols) are compared theoretical prediction (empty symbols). The Boltzmann distributions (dotted lines) are corrected by the photonic weights of the states. The $\emptyset 3 \mu \mathrm{m}$ and $\emptyset 9 \mu \mathrm{m}$ mesa emissions present thermalized distribution. The $\varnothing 20 \mu \mathrm{m}$ mesa emission has a nonthermal distribution. The vertical line is a separation between the LP0D and LP2D energy ranges.

(c) $\tau_{r} \ll \tau_{\text {ph }}$ : Polaritons recombine too fast to undergo enough scatterings with phonons. In this case, polaritons have a nonthermal distribution.

For the $\varnothing 9 \mu \mathrm{m}$ mesa, the effective temperature of $40 \mathrm{~K}$ suggests that thermalization becomes less efficient when the mesa diameter increases. This tendency is confirmed by the nonthermal emission obtained for the $\varnothing 20 \mu \mathrm{m}$ mesa.

In planar microcavities, Stanley et al. ${ }^{11}$ observed that for similar temperatures $(4-5 \mathrm{~K})$, polaritons did not thermalize. By increasing the lattice temperature up to $30 \mathrm{~K}$, they significantly shortened the polariton-phonon scattering time and could eventually observe a Boltzmann distribution. In our case, the lattice temperature remains unchanged but the confinement in small mesas has a crucial effect: the widening of the wave functions in the momentum space ${ }^{17}$ enhances the polariton-phonon scattering rate and thus favors thermalization in the small mesas. The comparison with theory is developed in Sec. VI.

\section{B. Dimensionality}

Observations (ii) and (iv) strongly suggest that, whereas the relaxation is very efficient within the localized states, it is suppressed between delocalized states and localized states. However, considering the low probability distribution of the delocalized states above the mesa, it is important to verify that (iv) is not the result of a poor mode matching between the excitation spot and the wave function of the delocalized polariton. We unambiguously confirmed this point with a cwPLE experiment (Fig. 5).

Thanks to the narrow spectral linewidth of the continuous-wave excitation. We were able to excite resonantly single polariton states. We could accurately measure, for each of them, the reflected and transmitted intensities in order to extract the absorption. We then normalized the emis-

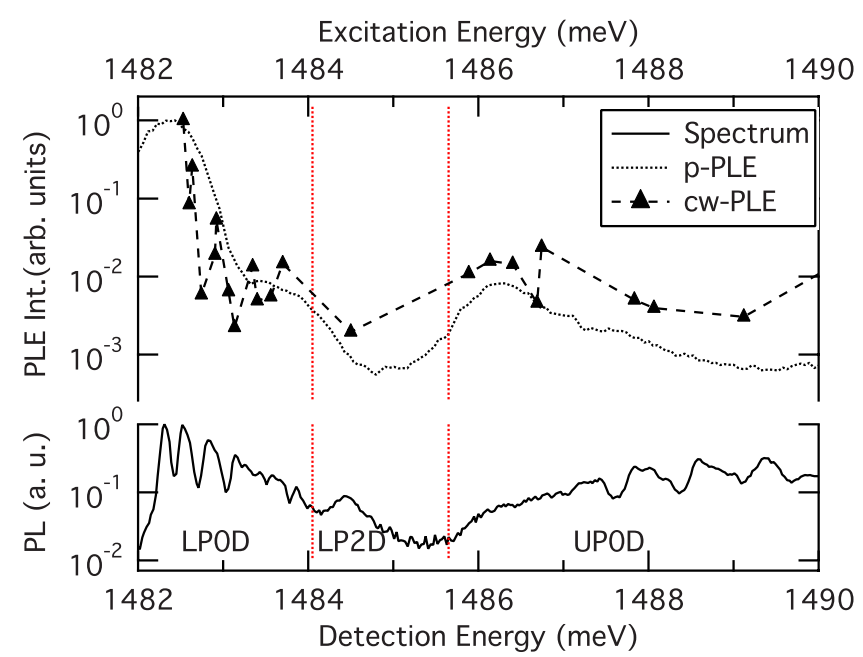

FIG. 5. (Color online) Comparison between the PLE spectra obtained with a pulse-shaped pump (dotted line) and with a cw pump (triangles). The dashed line is a guide for the eyes. The nonresonant photoluminescence spectrum (solid line) is plotted as a reference. The dotted lines are separations between the LP0D, LP2D, and UP0D energy ranges.

sion of the ground state by the absorption of the pumped state. The measured absorption of the LP2D was $15 \%$ less than the average absorption of the OD polaritons.

The results of the cw-PLE experiment on the 9- $\mu \mathrm{m}$-diameter mesa are displayed in Fig. 5, together with the p-PLE intensity horizontal profile taken at the groundstate energy of Fig. 3(b). For comparison, the figure also features a nonresonant excitation spectrum. One can notice the similar behavior of the p-PLE and the cw-PLE curves despite the fact that the latter is normalized. In both cases, the PLE intensity decreases by 1 order of magnitude when the excitation is resonant with the LP2D states. This demonstrates that the relaxation between $2 \mathrm{D}$ and $0 \mathrm{D}$ polaritons is inefficient.

Consequently, we can infer that under nonresonant excitation, the 2D states may not be an intermediate channel for the relaxation to the $0 \mathrm{D}$ states. The $0 \mathrm{D}$ states are most probably directly populated from the excitonic reservoir.

\section{RELAXATION DYNAMICS}

The dynamics of polariton relaxation has been investigated through TRPL experiments under resonant excitation. After exciting a given OD polariton state, we looked at the time-resolved evolution of the emission of the ground state. The luminescence was first sent to the spectrometer and then to a streak camera with 2 ps time resolution. Note that because of the dispersion on the grating, the experimental time resolution was about $10 \mathrm{ps}$. We focused our study on the 3- $\mu \mathrm{m}$ - and 9- $\mu \mathrm{m}$-diameter mesas, in which polaritons thermalize efficiently with the phonon bath.

In Figs. 6 and 7, we show the ground-state TRPL intensity for the $\varnothing 9 \mu \mathrm{m}$ and $\varnothing 3 \mu \mathrm{m}$ mesas, respectively. The luminescence intensity of the ground state increases with a rise time of about 15 ps. This time corresponds to the lifetime of 


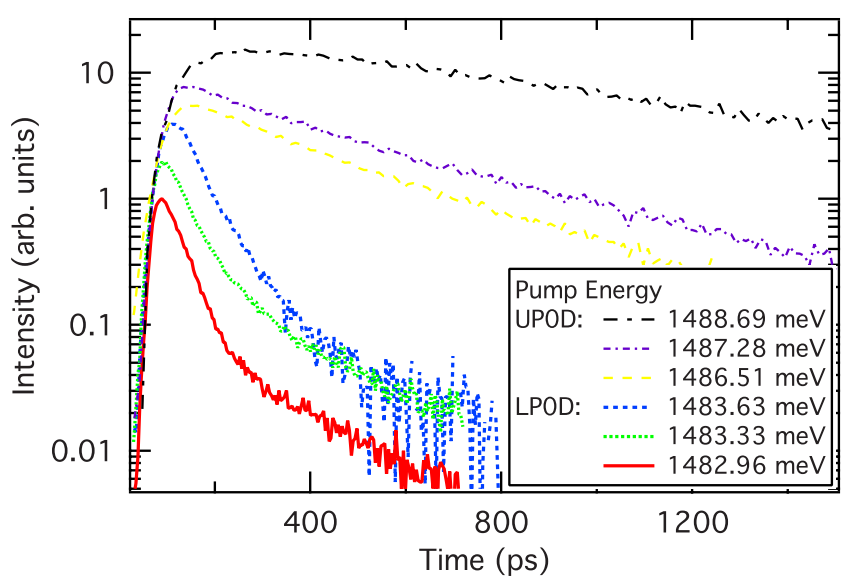

FIG. 6. (Color online) Time-resolved photoluminescence intensity of the ground state of a $9-\mu \mathrm{m}$-diameter mesa for different excitation energies. When the excitation energy is resonant with LP0D states, we clearly distinguish two relaxation times. When the excitation energy is resonant with UP0D states, there is only one decay time. For the sake of clarity, the curves have been arbitrarily displaced along the vertical axis.

the ground state. On the other hand, the decay times strongly depend on whether the excitation is resonant with a LPOD state or with a UPOD state.

For the $\emptyset 9 \mu \mathrm{m}$ mesa (Fig. 6), when the pump is resonant with a LPOD state, we observe a biexponential decay of the ground-state TRPL intensity. When the pump is resonant with a UP0D state, we observe a monoexponential decay. For the $\emptyset 3 \mu \mathrm{m}$ mesa (Fig. 7), the decay is monoexponential in both cases.

The values of the fitted decay times are reported in Table I for the $\emptyset 9 \mu \mathrm{m}$ mesa and in Table II for the $\emptyset 3 \mu \mathrm{m}$ mesa. We denote $\tau_{S}$ as the short decay time (less than 100 ps) and $\tau_{L}$ as the long decay time (more than $100 \mathrm{ps).}$

When the excitation energy is resonant with LP0D states, we obtain short decay times for both mesas. The values of $\tau_{S}$ vary between 3 and 5 times the lifetime of the ground state.

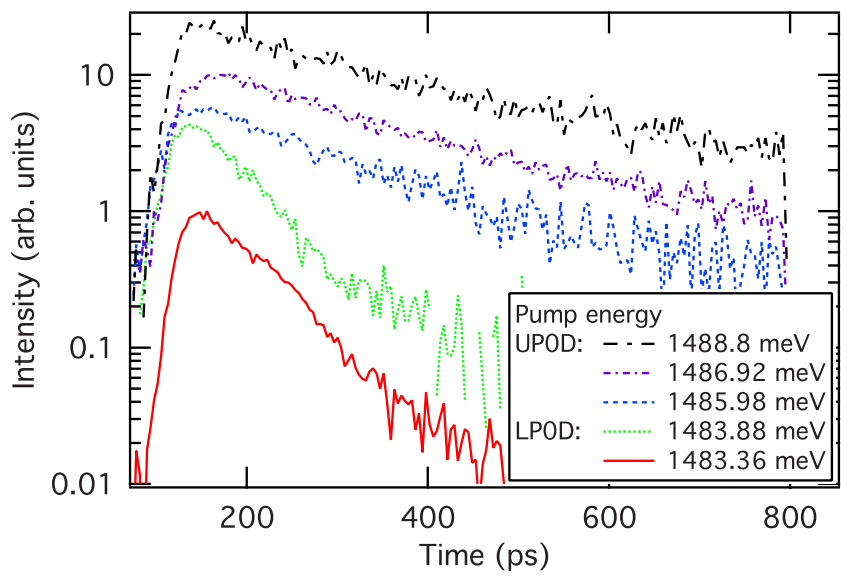

FIG. 7. (Color online) Time-resolved photoluminescence intensity of the ground state of a 3- $\mu$ m-diameter mesa for different excitation energies. Unlike Fig. 6, we observe a monoexponential decay at all excitation energies. For the sake of clarity, the curves have been arbitrarily displaced along the vertical axis.
TABLE I. Decay times of the ground-state TRPL intensity of a $\emptyset 9 \mu \mathrm{m}$ mesa for different excitation energies. We observe two decay times when exciting LPOD states and only one when exciting UP0D states.

\begin{tabular}{lccc}
\hline \hline & $\begin{array}{c}\text { Excitation energy } \\
(\mathrm{meV})\end{array}$ & $\begin{array}{c}\tau_{S} \\
(\mathrm{ps})\end{array}$ & $\begin{array}{c}\tau_{L} \\
(\mathrm{ps})\end{array}$ \\
\hline LP0D & 1482.96 & 45 & 134 \\
& 1483.33 & 50 & 137 \\
& 1483.63 & 55 & 137 \\
UP0D & & & \\
& 1486.51 & & 274 \\
& 1487.27 & & 320 \\
& 1488.69 & & 907 \\
\hline \hline
\end{tabular}

In the case of the $\varnothing 9 \mu \mathrm{m}$ mesa, we also obtain long decay times comparable to the decay times obtained while exciting UP0D states. The values of $\tau_{L}$ are 1-2 orders of magnitude longer than the lifetime of the ground state. These observations suggest that similar relaxation processes are responsible for the long decay times $\tau_{L}$ observed in both mesas.

A possible phenomenological interpretation of these observations is the following:

(a) The relaxation of polaritons created in the UPOD involves polariton states with large excitonic content (LXC). Their long radiative lifetime determines the long decay time of the ground-state TRPL intensity while exciting UPOD states.

(b) Considering the interactions with the phonon bath, polaritons created in a LPOD state have two relaxation paths to the ground state: a direct path, to lower-energy states, and an indirect path, corresponding to an initial scattering to states of higher energy followed by relaxation. This latter path possibly gives rise to a second decay time in the ground-state luminescence intensity.

(c) In the case of the $\varnothing 3 \mu \mathrm{m}$ mesa, the observation of a monoexponential decay while exciting LP0D states suggests that the indirect relaxation path is less efficient than in the $\varnothing$ $9 \mu \mathrm{m}$ mesa. This could be due to the larger energy separation of LPOD states in $\varnothing 3 \mu \mathrm{m}$ mesas.

The theory exposed in Sec. V provides a comprehensive and quantitative theoretical description of our results.

TABLE II. Decay times of the ground-state TRPL intensity of a Ø $3 \mu \mathrm{m}$ mesa for different excitation energies. We observe only one decay time at each excitation energy.

\begin{tabular}{lccc}
\hline \hline & $\begin{array}{c}\text { Excitation energy } \\
(\mathrm{meV})\end{array}$ & $\begin{array}{c}\tau_{S} \\
(\mathrm{ps})\end{array}$ & $\begin{array}{c}\tau_{L} \\
(\mathrm{ps})\end{array}$ \\
\hline LP0D & 1483.36 & 71 & \\
& 1483.88 & 59 & \\
& & & \\
UP0D & 1485.98 & & 172 \\
& 1486.92 & & 183 \\
& 1488.80 & & 219 \\
\hline \hline
\end{tabular}




\section{THEORETICAL MODEL}

The theoretical analysis is performed in two steps. First, we compute the polariton quasimodes for the present nonuniform case. ${ }^{18}$ Second, we map the polariton-phonon coupling into the basis of the obtained polariton quasimodes. This procedure is justified by the fact that, in spite of the present nonequilibrium regime, the quasiparticle approximation still holds, as discussed below.

\section{A. Polariton quasimodes}

Polariton quasimodes can be computed via Maxwell equations, as in Ref. 18, or by solving a nonequilibrium Schrödinger-type equation for the exciton-photon problem. ${ }^{20,21}$ The spectrum of excitation, i.e., the frequencies and the lifetimes, obtained within the two approaches is substantially the same, with minor quantitative differences. This quantitative agreement makes the quasiparticle approximation suitable to describe the present nonequilibrium regime.

The exciton and photon Bose fields $\hat{\Psi}_{x, c}(\mathbf{r})$ can then be safely written in terms of the polariton quasimodes as

$$
\hat{\Psi}_{x, c}(\mathbf{r})=\sum_{j, n} \psi_{x, c}^{j, n}(\mathbf{r}) \hat{p}_{j, n},
$$

where the label $j$ defines the quantum number of the mode and the label $n=\mathrm{LP}$, UP identifies the lower and the upper polariton branches, while $\hat{p}_{j, n}$ is the polariton annihilation operator obeying Bose statistics. Obviously the sum in Eq. (1) refers to both localized (0D) and delocalized (2D) modes. Let us remind here that the exciton and photon wave functions $\psi_{x, c}^{j, n}(\mathbf{r})$ have the same spatial profile, while their amplitudes depend, respectively, on the excitonic and photonic contents of the corresponding polariton mode.

\section{B. Coupling to acoustic phonons}

By means of the decomposition (1), the Hamiltonian describing the coupling between the exciton field and the bath of acoustic phonons ${ }^{13}$ can be written in the basis of polariton quasimodes as

$$
\hat{H}_{X \mathrm{ph}}=\sum_{j, n, j^{\prime}, n^{\prime}, \mathbf{q}} t_{(j, n),\left(j^{\prime}, n^{\prime}\right)}^{\mathbf{q}}\left(\hat{a}_{\mathbf{q}}+\hat{a}_{-\mathbf{q}}^{\dagger}\right) \hat{p}_{j, n}^{\dagger} \hat{p}_{j^{\prime}, n^{\prime}} .
$$

The matrix elements $t_{(j, n),\left(j^{\prime}, n^{\prime}\right)}^{\mathbf{q}}$ involve the excitonic wave functions and are given by ${ }^{22}$

$$
t_{(j, n),\left(j^{\prime}, n^{\prime}\right)}^{\mathbf{q}}=C_{\mathbf{q}} \sqrt{\frac{\hbar \omega_{\mathbf{q}}^{\mathrm{ph}}}{A}} \int d \mathbf{r} \psi_{x}^{j, n^{*}}(\mathbf{r}) e^{-i \mathbf{q}_{\|} \cdot \mathbf{r}^{\prime}} \psi_{x}^{j^{\prime}, n^{\prime}}(\mathbf{r}),
$$

where

$$
C_{\mathbf{q}}=\frac{\left[D_{e} K_{e}\left(q_{z}\right) \chi\left(\mathbf{q}_{\|} \eta_{e}\right)+D_{h} K_{h}\left(q_{z}\right) \chi\left(\mathbf{q}_{\|} \eta_{\mathbf{h}}\right)\right]}{\sqrt{2 u_{s}^{2} \rho_{M} L_{c}}},
$$

$A$ is the phonon quantization area, $u_{s}$ is the sound velocity, $\rho_{M}$ is the mass density, $L_{c}$ is the cavity length, $D_{e, h}$ are the deformation potentials, $\eta_{e, h}=\left(m_{e}+m_{h}\right) / m_{h, e}, \quad \omega_{\mathbf{q}}^{p h}=u_{s}\left(q_{z}^{2}\right.$ $\left.+\mathbf{q}_{\|}^{2}\right)^{1 / 2}$ is the frequency, $q_{\|}$is the in-plane wave vector of the phonon mode $\mathbf{q}$, and, for Gauss confinement and hydrogenlike electron-hole relative motion, $K_{e, h}\left(q_{z}\right)$ $=\exp \left[-\left(q_{z} L_{e, h}\right)^{2} / 2\right]$ and $\chi\left(\mathbf{q}_{\|}\right)=\left[1+\left(q_{\|} a_{0} / 2\right)^{2}\right]^{3 / 2}$.

Accounting for the phonon coupling within the Markovian approximation, and for the finite lifetime, the rate equations for the populations $N_{j, n}=\left\langle\hat{p}_{j, n}^{\dagger} \hat{p}_{j, n}\right\rangle$ in the different polariton states $(j, n)$ are ${ }^{13,22}$

$$
\begin{aligned}
\dot{N}_{j, n}= & -\Gamma_{j, n} N_{j, n}+\sum_{j^{\prime}, n^{\prime}} W_{\left(j^{\prime}, n^{\prime}\right),(j, n)} N_{j^{\prime}, n^{\prime}}\left(N_{j, n}+1\right)-W_{(j, n),\left(j^{\prime}, n^{\prime}\right)} \\
& \times\left(N_{j^{\prime}, n^{\prime}}+1\right) N_{j, n},
\end{aligned}
$$

where the phonon-scattering rates are ${ }^{13,22}$

$$
\begin{aligned}
W_{(j, n),\left(j^{\prime}, n^{\prime}\right)}= & \sum_{\mathbf{q}}\left|t_{(j, n),\left(j^{\prime}, n^{\prime}\right)}^{\mathbf{q}}\right|^{2}\left[n_{B}\left(\left|\Delta E_{j^{\prime}, n^{\prime}}^{j, n}\right|\right)\right. \\
& \left.+\theta\left(\Delta E_{j^{\prime}, n^{\prime}}^{j, n}\right)\right] \delta\left(\hbar \omega_{\mathbf{q}}^{\mathrm{ph}}-\left|\Delta E_{j^{\prime}, n^{\prime}}^{j, n}\right|\right),
\end{aligned}
$$

$\Delta E_{j^{\prime}, n^{\prime}}^{j, n}=E_{j, n}-E_{j^{\prime}, n^{\prime}}$ is the energy difference between the states $(j, n)$ and $\left(j^{\prime}, n^{\prime}\right), n_{B}(E)$ is the Bose distribution, while $\Gamma_{j, n}$ are the effective polariton lifetimes. These quantities are consistently obtained from the diagonalization of the exciton-photon problem.

Notice that in Eq. (6) the sum spans over all the momentum states of phonons, i.e., $q_{z}=\left(2 \pi / L_{c}\right) n_{z}$ and

$$
q_{x, y}=\frac{2 \pi}{\sqrt{A}} n_{x, y}
$$

with $n_{x, y, z}=0,1, \ldots$ and where $A$ is the phonon quantization area also appearing in Eq. (3). In the uniform case, for each couple of initial and final states, the only contribution is due to phonon modes having in-plane momentum equal to the momentum difference between the two states. ${ }^{13}$ Contrarily, in the trapped case, for two polariton states localized in a much smaller area $A_{\text {mesa }} \ll A$ [i.e., wave functions $\psi_{x}^{j, n}(\mathbf{r})$ are localized within $A_{\text {mesa }}$, it is easy to prove that all the phonon modes with in-plane momentum comprised in the interval $\Delta q_{x} \times \Delta q_{y}=4 \pi^{2} / A_{\text {mesa }}$ around $\mathbf{q}$ equally contribute. This means that, for each couple of localized states, the number of phonon modes contributing to the relaxation is proportional to the inverse of $A_{\text {mesa }}$. Then we expect that the relaxation rates scale as $W \sim 1 / A_{\text {mesa }}$.

On the other hand, the matrix element involving one delocalized and one localized polariton modes is given by Eq. (3) where one wave function is a plane wave,

$$
t_{(j, n), 2 \mathrm{D}}^{\mathbf{q}} \propto \int_{A_{\mathrm{mesa}}} d \mathbf{r} u_{x}^{j, n *}(\mathbf{r}) e^{-i \mathbf{q}_{\|} \cdot \mathbf{r}} \frac{e^{i \mathbf{k}_{2 \mathrm{D}} \cdot \mathbf{r}}}{\sqrt{A_{2 \mathrm{D}}}} .
$$

Here, $A_{\text {mesa }}$ and $A_{2 \mathrm{D}}$ are the quantizing surfaces for the mesa state and for the delocalized state, respectively.

For the ground state, $u_{x}^{0, \mathrm{LP}} \sim 1 / \sqrt{A_{\text {mesa }}}$ and the contributing phonon modes have wave vectors $\mathbf{q}_{\|} \sim \mathbf{k}_{2 \mathrm{D}}$. The corresponding matrix elements are proportional to $\sqrt{A_{\text {mesa }} / A_{2 \mathrm{D}}}$.

For the calculations, we use a confining potential in agreement with the atomic force microscope (AFM) images of the mesas, ${ }^{16}$ thus accounting for the slightly elliptical shape of the mesas. The decay rates are assumed to be $\gamma_{c}$ $=0.05 \mathrm{meV}$, in agreement with the reported photon lifetime $\tau_{c}=15 \mathrm{ps}$, and $\gamma_{x}=0.001 \mathrm{meV}$. The other parameters are ob- 


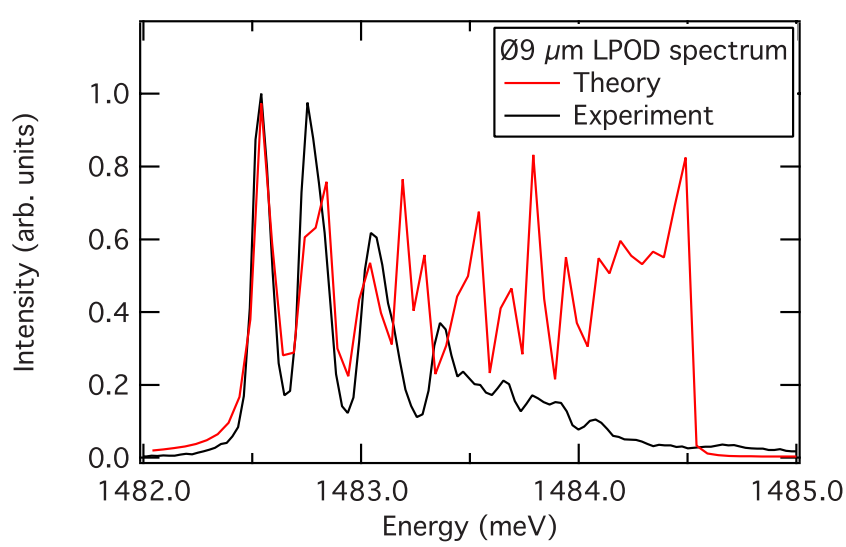

FIG. 8. (Color online) Theoretical and experimental spectra of the LPOD under nonresonant excitation. No relaxation process is taken into account in the plotted theoretical spectrum.

tained from the experimental characterization of the sample.

The polariton quasimodes computation also accounts for the presence of two-body interactions, whose effect could be in principle very important and amplified by the reduced sizes. We have theoretically verified that, for the pump intensities used in this experiment, the system can be considered in the linear regime, with the two-body interactions becoming relevant and eventually dominating only for pump intensities of 2-3 orders of magnitude larger.

\section{DISCUSSION}

We now compare the theoretical predictions about polaritons modes and relaxation to the experimental results presented above. The thermalization efficiency and the influence of dimensionality are discussed. Finally, the time-dependent behavior of the relaxation is evaluated.

\section{A. Polariton spectrum}

The calculated luminescence spectrum for the LPOD is plotted in Fig. 8, together with an experimental spectrum. One can appreciate the correspondence of the observed and computed energies and linewidths. The theoretical spectrum is weighted by the photonic content of the states but does not take any relaxation mechanism into account. The strong intensity difference between theory and experiment for the excited states is a clear indication of the important role of relaxation mechanisms.

The energies of the polariton states obtained theoretically are in good agreement with the experiment and can therefore be used to simulate the relaxation between different states.

\section{B. Thermalization}

The relaxation is accounted for by applying the rate equations [Eq. (5)] to the predicted modes. These rate equations depend on the phonon-scattering rates. Note that the scattering rates to states of higher energy decrease exponentially with the energy difference between the initial and final polariton states [see Eq. (6)]. On the other hand, the scattering

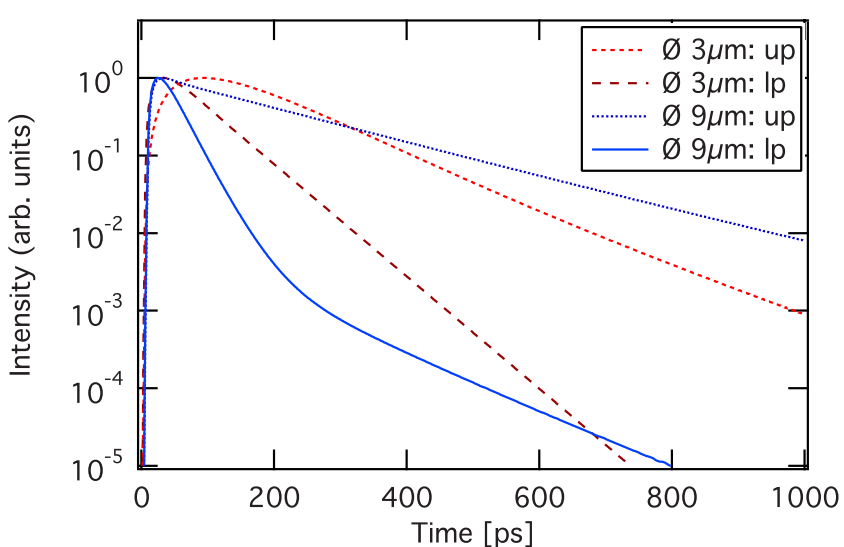

FIG. 9. (Color online) Calculated TRPL of the ground state in a $\varnothing 3 \mu \mathrm{m}$ mesa and a $\varnothing 9 \mu \mathrm{m}$ mesa when exciting resonantly the LPOD or the UP0D states. The behaviors of Figs. 6 and 7 are well reproduced by the theory. Two decay times are predicted in the relaxation of a $\varnothing 9 \mu \mathrm{m}$ mesa LPOD state.

rates to lower-energy states always have a spontaneous contribution coming from the Heaviside function $\theta$.

In order to reproduce the thermalization experiments presented in Sec. III, we computed the relaxation dynamics after resonant excitation of a UP0D state. The thermalization dependence on the mesa diameter is accurately reproduced, as shown in Fig. 4. The predicted effective temperatures are the same as the one obtained experimentally: 15 and $40 \mathrm{~K}$ for the $3-\mu \mathrm{m}$ - and 9- $\mu \mathrm{m}$-diameter mesas, respectively.

As explained in Sec. V, the scattering rate $W$ between two OD states is inversely proportional to the area of the mesa $1 / A_{\text {mesa }}[$ Eqs. (3) and (6)]. This explains the more efficient thermalization in the $\varnothing 3 \mu \mathrm{m}$ mesas.

The scattering rate between $2 \mathrm{D}$ and $0 \mathrm{D}$ states is predicted to be proportional to $\sqrt{A_{\text {mesa }} / A_{2 D}}$ [Eq. (8)]. In our case, this ratio is about 0.1 , assuming $A_{2 \mathrm{D}} \simeq 1000 \mu \mathrm{m}^{2} .{ }^{23}$ Therefore, the calculation gives a OD-OD coupling that is nearly 1 order of magnitude greater than the $2 \mathrm{D}-0 \mathrm{D}$ coupling. This is consistent with the results of Fig. 5.

\section{Dynamics}

The last comparison concerns the time-resolved photoluminescence experiments. By solving Eq. (5), we obtain the time evolution of the population in each polariton state.

The experimental time evolution of the ground-state emission intensity is displayed in Figs. 6 and 7. In Fig. 9 we plot the calculated time evolution of the population in the ground state for the two mesa sizes and for excitations in the LP0D and UPOD. The theory reproduces well the experiments. In particular we obtain two decay times in the case of the $\varnothing$ $9 \mu \mathrm{m}$ mesa when exciting resonantly a LP0D state.

The agreement is also quantitative. Decay rates have been extracted from the decay curves and the theoretical and experimental values are compared in Fig. 10. Here, the rates are plotted as a function of the excitation energy. The short decay times give rise to a fast decay regime, with rates between $10^{-2}$ and $2 \times 10^{-2} \mathrm{ps}^{-1}$. The long decay times give rise to a slow decay regime, with rates between $2 \times 10^{-3}$ and 5 


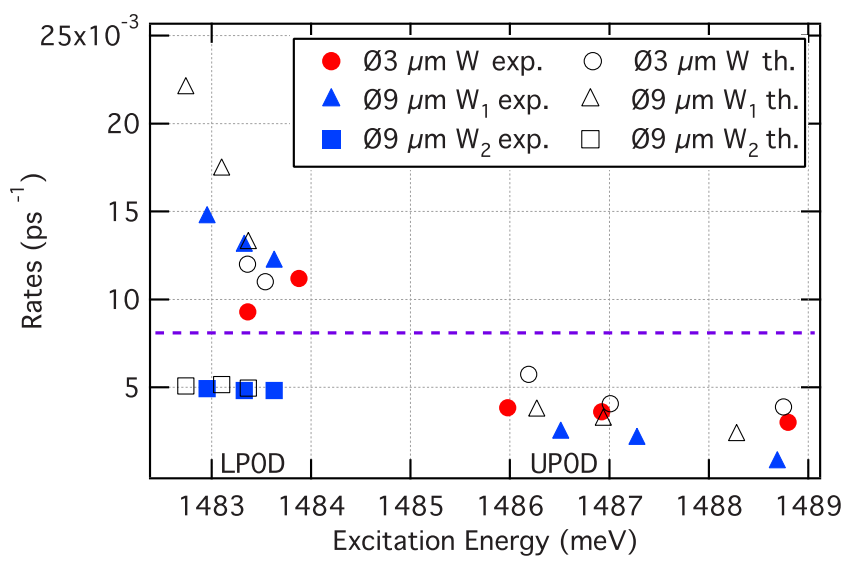

FIG. 10. (Color online) Decay rates in the $\varnothing 3 \mu \mathrm{m}$ mesa and in the $\varnothing 9 \mu \mathrm{m}$ mesa (full symbols) and comparison with theory (empty symbols). The data points at lower/higher energy than 1485 $\mathrm{meV}$ correspond to excitations resonant with LP0D/UP0D states. The dashed line separates the different decay regimes.

$\times 10^{-3} \mathrm{ps}^{-1}$. In Sec. VI D, we explain this trend by the fact that confined polariton states with LXC states participate in the relaxation process, as it has been proposed at the end of Sec. IV.

\section{Relaxation between localized states}

We now discuss how LXC states are responsible for the slow decay regime. As already discussed (Fig. 2 and Sec. II), the states close in energy to the exciton resonance have a small photonic content, i.e., a long radiative lifetime. They are involved in the relaxation paths from the excited states to the ground state, thus fixing the decay times observed experimentally for both excitations of UP0D and LP0D states.

\section{Excitation of UPOD states}

When exciting UP0D states, both $\emptyset 3 \mu \mathrm{m}$ and $\varnothing 9 \mu \mathrm{m}$ mesas present only a slow decay regime. The relaxation of UPOD states to the ground state requires many polaritonphonon scatterings. This results from Eq. (6), which asserts that the relaxation is favored between polariton states with small energy difference. Therefore, the relaxation of UPOD states has a very high probability to involve LXC states. These states have a radiative lifetime $\tau_{\text {LXC }}$ much longer that the one of the ground state $\tau_{\mathrm{GS}}$. This is represented in Fig. 11(a).

Due to the very efficient interactions with the phonon bath [cf. Eqs. (6) and (3)] and to the efficient thermalization observed in $\emptyset 3 \mu \mathrm{m}$ and $\emptyset 9 \mu \mathrm{m}$ mesas, we have the relation $\tau_{\mathrm{ph}} \leq \tau_{\mathrm{GS}}<\tau_{\mathrm{LXC}}$. In this case, as sketched in Fig. 11(a), the rise time of the ground-state luminescence intensity corresponds to $\tau_{\mathrm{GS}}$. Its decay reflects the population decay in the states with large excitonic content, i.e., $\tau_{\mathrm{LXC}}$. This leads to the observation of small decay rates in Fig. 10. The p-PLE experiment (Fig. 3) show that the number of LXC states increases with the diameter of the mesa. We thus expect a slower decay regime in the $\varnothing 9 \mu \mathrm{m}$ mesa than in the $\varnothing$ $3 \mu \mathrm{m}$ mesa. This difference can be appreciated in Fig. 10.

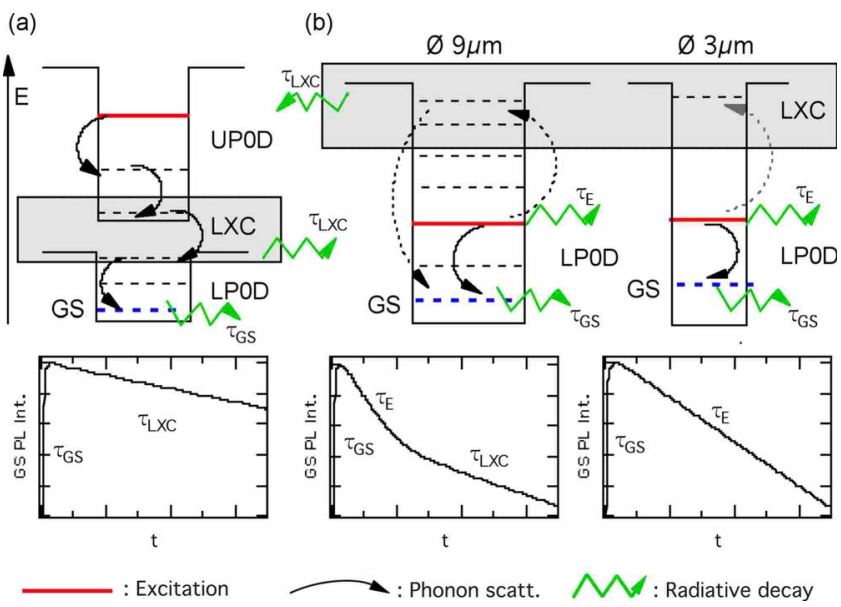

FIG. 11. (Color online) Scheme of the role of LXC states in polariton relaxation to the GS. (a) The excitation is resonant with a UP0D state. The relaxation necessarily involves LXC states. We observe only one decay time in the TRPL intensity of the ground state. (b) The excitation is resonant with a LPOD state. In a $\varnothing 9 \mu \mathrm{m}$ mesa, two relaxation paths are possible, giving rise to two decay times in the ground-state TRPL intensity. In case of a $\varnothing 3 \mu \mathrm{m}$ mesa, the scattering to LXC states is inefficient because of the too large energy separation between the localized states. In the bottom panels, we plot the TRPL intensity of the ground state as predicted by theory.

\section{Excitation of LPOD states}

When exciting LP0D states, we observe two decay regimes for the $\varnothing 9 \mu \mathrm{m}$ mesa and only one for the $\varnothing 3 \mu \mathrm{m}$ mesa. Due to the very efficient coupling to the phonon bath and to the finite temperature $(T>0)$, the thermalization process of the LP0D states also populates LXC states. Therefore we must consider two possible relaxation paths to the ground state, as represented in Fig. 11(b). The direct relaxation path manifests itself in a fast decay regime, whereas the indirect relaxation path, involving LXC states, manifests itself in a slow decay regime. Again, this contribution is more important for $\emptyset 9 \mu \mathrm{m}$ mesas than for $\emptyset 3 \mu \mathrm{m}$ mesas because of the larger number of LXC states available. Moreover, the energy separations between $\emptyset 9 \mu \mathrm{m}$ mesa levels are smaller compared to the lattice thermal energy $\left(k_{B} T \simeq 0.5 \mathrm{meV}\right)$. As a result, we do not observe a slow decay regime in the $\varnothing$ $3 \mu \mathrm{m}$ mesa.

\section{CONCLUSION}

We present experimental and theoretical results of polariton relaxation in mesa structures of different diameters. We observe an enhancement of the thermalization process, arising from the lateral confinement. The relaxation is favored between polaritons of the same dimensionality, suggesting that under nonresonant excitation, the $0 \mathrm{D}$ polaritons are populated more efficiently from the exciton reservoir than from the $2 \mathrm{D}$ polariton states.

The relaxation dynamics is accessed by time-resolved photoluminescence experiments. We extract characteristic rates and evidence the role of polariton states with large ex- 
citonic content in the relaxation between localized levels.

A theoretical model based on semiclassical rate equations reproduces accurately the luminescence spectrum and the dynamics of the relaxation in the low-density regime. It provides clear and comprehensive understanding of the thermalization and its dependence on dimensionality. The quantitative agreements between theory and experiment allows identifying polariton-phonon scattering as the major relaxation process.

The results of this paper show that polariton mesas are the ideal system to study the thermalized polariton gas at low temperatures $(T<5 \mathrm{~K})$. This is a key ingredient to characterize BEC and superfluidity because it allows the thermal control of fluctuations in the system.

\section{ACKNOWLEDGMENTS}

The authors thank S. Portolan and M. Wouters for fruitful discussions and A. Galler and Th. Feurer for useful advice on ultrafast pulse shaping. The present work was supported by the Swiss National Research Foundation through "NCCR Quantum Photonics.” *taofiq.paraiso@epfl.ch

${ }^{\dagger}$ Present address: Institut Néel-CNRS 25 Avenue des Martyrs, BP 166 Fr-38042 Grenoble Cedex 9, France.

†Present address: INL, UMR CNRS 5270, Ecole Centrale de Lyon

36, Av. Guy de Collongue, 69134 Ecully Cedex, France.

${ }^{1}$ J. Kasprzak et al., Nature (London) 443, 409 (2006).

${ }^{2}$ R. Balili, V. Hartwell, D. Snoke, L. Pfeiffer, and K. West, Science 316, 1007 (2007).

${ }^{3}$ H. Deng, G. S. Solomon, R. Hey, K. H. Ploog, and Y. Yamamoto, Phys. Rev. Lett. 99, 126403 (2007).

${ }^{4}$ D. Bajoni, P. Senellart, E. Wertz, I. Sagnes, A. Miard, A. Lemaitre, and J. Bloch, Phys. Rev. Lett. 100, 047401 (2008).

${ }^{5}$ G. Christmann, R. Butté, E. Feltin, J.-F. Carlin, and N. Grandjean, Appl. Phys. Lett. 93, 051102 (2008).

${ }^{6}$ P. G. Savvidis, J. J. Baumberg, R. M. Stevenson, M. S. Skolnick, D. M. Whittaker, and J. S. Roberts, Phys. Rev. Lett. 84, 1547 (2000).

${ }^{7}$ A. Griffin, D. W. Snoke, and S. Stringari, Bose-Einstein Condensation (Cambridge University Press, Cambridge, 1995).

${ }^{8}$ K. G. Lagoudakis, M. Wouters, M. Richard, A. Baas, I. Carusotto, R. André, L. S. Dang, and B. Deveaud-Plédran, Nat. Phys. 4, 706 (2008).

${ }^{9}$ D. Sarchi and V. Savona, Phys. Rev. B 77, 045304 (2008).

${ }^{10}$ F. M. Marchetti, M. H. Szymanska, J. M. J. Keeling, J.
Kasprzak, R. Andre, P. B. Littlewood, and L. Si Dang, Phys. Rev. B 77, 235313 (2008).

${ }^{11}$ R. P. Stanley, R. Houdré, C. Weisbuch, U. Oesterle, and M. Ilegems, Phys. Rev. B 53, 10995 (1996).

${ }^{12}$ M. Perrin, P. Senellart, A. Lemaitre, and J. Bloch, Phys. Rev. B 72, 075340 (2005).

${ }^{13}$ F. Tassone, C. Piermarocchi, V. Savona, A. Quattropani, and P. Schwendimann, Phys. Rev. B 56, 7554 (1997).

${ }^{14}$ D. Sarchi and V. Savona, Phys. Rev. B 75, 115326 (2007).

${ }^{15}$ D. Bajoni, E. Peter, P. Senellart, J. L. Smirr, I. Sagnes, A. Lemaître, and J. Bloch, Appl. Phys. Lett. 90, 051107 (2007).

${ }^{16}$ O. El Daïf, A. Baas, T. Guillet, J.-P. Brantut, R. I. Kaitouni, J. L. Staehli, F. Morier-Genoud, and B. Deveaud, Appl. Phys. Lett. 88, 061105 (2006).

${ }^{17}$ R. I. Kaitouni et al., Phys. Rev. B 74, 155311 (2006).

${ }^{18}$ V. Savona, J. Phys.: Condens. Matter 19, 295208 (2007).

${ }^{19}$ H. Deng, D. Press, S. Gotzinger, G. S. Solomon, R. Hey, K. H. Ploog, and Y. Yamamoto, Phys. Rev. Lett. 97, 146402 (2006).

${ }^{20}$ I. Carusotto and C. Ciuti, Phys. Rev. B 72, 125335 (2005).

${ }^{21}$ D. Sarchi, M. Wouters, and V. Savona, arXiv:0811.2721 (unpublished).

${ }^{22}$ G. Mannarini and R. Zimmermann, Phys. Rev. B 73, 115325 (2006).

${ }^{23}$ W. Langbein and J. M. Hvam, Phys. Rev. Lett. 88, 047401 (2002). 